\title{
Construction of evolutionary mathematical model of hierarchical network topology
}

\author{
Min Yang, \\ Dean's Office, Chongqing City Vocational College, \\ Chongqing, 402160 \\ China
}

Received: March 3, 2021. Revised: August 12, 2021. Accepted: August 28, 2021. Published: August 31, 2021.

\begin{abstract}
In order to solve the problem that the traditional hierarchical network topology evolution mathematical model has low accuracy in describing the dynamic behavior of network, the design of hierarchical network topology evolution mathematical model is proposed. This paper analyzes the hierarchical network, establishes the effectiveness index of topology, formulates the strategy of topology reconstruction, realizes the evolution of hierarchical network topology, and completes the design of mathematical model. The experimental results show that the accuracy of the designed mathematical model of hierarchical network topology evolution can reach $94 \%$, and the effect is good in practical application.
\end{abstract}

Keywords-Blockchain technology, logistics big data, shared value, visualization platform.

\section{INTRODUCTION}

$\mathrm{T}$ HE Internet is becoming more and more inseparable from people's daily work and life. Due to its large scale, complex structure, decentralized management, and changeable status, it still lacks effective network topology detection technologies and models to accurately describe the Internet topology. But the research on modeling of Internet network topology has never stopped. The network is a network structure composed of a set of intersecting nodes and lines, and the network model is a network that reflects a complete system. The network model is not only a graphical model, it can not only reflect the relationship between various elements and parameters, but also can be analyzed and calculated according to a certain topological logic relationship. As a standard model, it can give the best answer to a question under certain conditions, and it can also provide the progress of an action or plan. This is the most widely used, most common and ideal model in scientific research, engineering technology, production technology, and management. Many scientific theories are the standard models that study how to build and solve various problems and systems more effectively. For example, the linear programming model is a widely used model. However, most models are only suitable for a specific or specialized problem at the beginning of the research phase, and cannot be widely used as standard models. With the continuous summary and in-depth study of the model application, it gradually developed into a standard model. Network models can reflect both positive and indefinite problems. In a broad sense, the positive network model is just a special case of a random network model. Network models can also reflect dynamic problems. The direction of the arrows (branch lines, arrow lines) in the network model reflects the progress and development of the problem. It can be seen that the network model is a comprehensive standard model and can be widely used in many aspects.

Computer network systems are layered to build a layered network. Each layer has its own function, and the layers are different according to different standards. For example, the hierarchical structure of the OSI (Open System Interconnection) standard consists of the physical layer, data link layer, network layer, transport layer, session layer, presentation layer, and application layer in the order from bottom to top. TCP / IP (Transmission Control Protocol / Internet Protocol) standards are host-network layer, network interconnection layer, transport layer, and application layer. These hierarchies are implemented through protocols. For example, the physical layer products have interfaces for transmission media, the data link layer products include network cards and bridges, and the network layer products include routers and gateways. The typical models that were initially applied to the evolutionary modeling of the hierarchical network topology are the Waxman model and the Transit-Stub model [1] [2]. These two models construct random networks based on the probability of connection between nodes and construct hierarchical networks according to the principle of hierarchy. Some researchers have proposed evolutionary algorithm for network topology design, which provides an appropriate mathematical framework through fuzzy logic, 
avoids the uncertainty of network traffic and other design parameters, and optimizes multiple objectives [3]. Some scholars have proposed a hierarchical network model of network topology design based on genetic algorithm. The hierarchical network model is composed of the upper logic circuit. Combined with its own similarity, the traffic information switch model is used to establish the connection with the network equipment to obtain the minimum network constraints [4]. As the research progressed, it was found that none of these models could well reflect the network characteristics of the Internet. Although these models better simulate some of the characteristics of hierarchical networks, they all have their own shortcomings. Compared with the statistical parameters of the real Internet, they appear to have insufficient accuracy.

Therefore, the evolutionary mathematical model of hierarchical network topology is designed. Using the actual characteristics of the Internet and the theory of preference growth, the hierarchical structure in the Transit-Stub model is combined with the characteristics of preference growth in the BA model (Barabási-Albertmodel). Considering factors such as node growth and preferential connection, the growth and demise of internal and external links between nodes, the hierarchical network topology evolution model is constructed.

The rest of this paper is organized as follows.

Section 2: This paper introduces the composition and characteristics of hierarchical network.

Section 3: The paper analyzes the topological validity index, proposes the strategy of topology reconstruction, and establishes an evolutionary mathematical model of hierarchical network topology.

Section 4: The platform of experiment and the analysis of experimental results prove the effectiveness of the model design.

Section 5: This paper makes a comprehensive summary of the article, puts forward the design significance of the design model, and puts forward the future research and development direction and suggestions.

\section{Materials AND Methods}

Hierarchical networks represent a large network plan in the form of a layered network plan. It can be obtained by decomposing step by step from the whole to the part, or it can be formed by gradual merging and simplification of the subordinate network [5]. The lower layer network can be simplified into one or several processes in the upper layer network. According to the principles of system engineering, any system has separable characteristics regardless of its complicated program. The total system can be decomposed into several secondary subsystems, and each secondary subsystem can be decomposed into three subsystems. Different standards give different subsystems for decomposition. This simplifies complex systems.

For the network graph plan, it can be decomposed into sub-networks (or merged into a total network gradually) according to certain hierarchical standards (principle), and then the connections between the layers of the network are determined, and a time parameter algorithm is proposed. Finally, multi-layer networks are effectively processed using network planning technology. Large or complex projects have as many as hundreds, thousands, or even tens of thousands of procedures, and they must use enough work to express their logical relationships and facilitate management and control. In this case, it is necessary to represent the project in a hierarchical manner. If the network is not hierarchical, and only one network is used to represent a larger or complex project, there are some disadvantages:

First: It takes a long time to modify, adjust and update the network;

Second: When working too much (up to several hundred roads), the network diagram is not easy to control;

Third: It is difficult for people who lack experience in project planning to control and manage the project. The method of layering the network can not only avoid these disadvantages, but also have the following advantages:

First, with the accumulation of experience and deepening of project understanding, network maps with different levels of details can be gradually prepared;

Second, the second-level network can be easily refined to the third-level network, making it easy for project implementers to clarify their work goals;

Third, it is easy to understand, update and adjust after the network is classified;

Fourth, the tasks of updating, adjusting, and reporting can be directly completed by the specific person responsible for improving accuracy and work efficiency.

Large-scale network plans have tens of thousands of nodes, and there are few division levels. This results in too many and complicated nodes per network, and it is very difficult to implement and control the plan. If the decomposition is not detailed enough, the key points will not be exposed. The subsequent system analysis will be futile, and it is easy to miss the opportunity to find an optimal solution. Conversely, if there are too many levels of division, it is necessary to deal with the complex relationships between many layers of networks, and the network diagrams will increase dramatically, the amount of calculations will increase exponentially, and it will also increase the trouble for compilers [6]. It is best to divide a level that is practical and convenient. In practice, we find that the three levels are better levels, which are suitable for large and medium-sized projects.

Features of a layered network:

First, the sub-network union is a subset of the overall network, which means that some activities of the upper network are not subdivided;

Second, each sub-network can have several starting points and several end points, which are reflected in the upper-level network, and there are several road lines corresponding to it. The length of each active route corresponds to the length of some key routes in the sub-network [7].

Third, nodes in the same layer may have nodes overlapping, 
but no activity overlapping.

Fourth, in the decomposition process, the same node can be allocated to different sub-networks.

There is a phenomenon that regional connections are preferred in real networks. Hierarchical networks have hierarchical relationships such as regional connections and international interconnection at the AS level. New AS is usually only connected to other AS that is adjacent to each other in the region. They are often tightly connected within the regional network. When the new AS joins the network, it does not give priority to linking with the entire network, but first chooses to link with nodes with the high degree of node. Therefore, the two-level regional network model defines a hierarchical network: the WAN and the LAN, as shown in Fig. 1.

Hierarchical

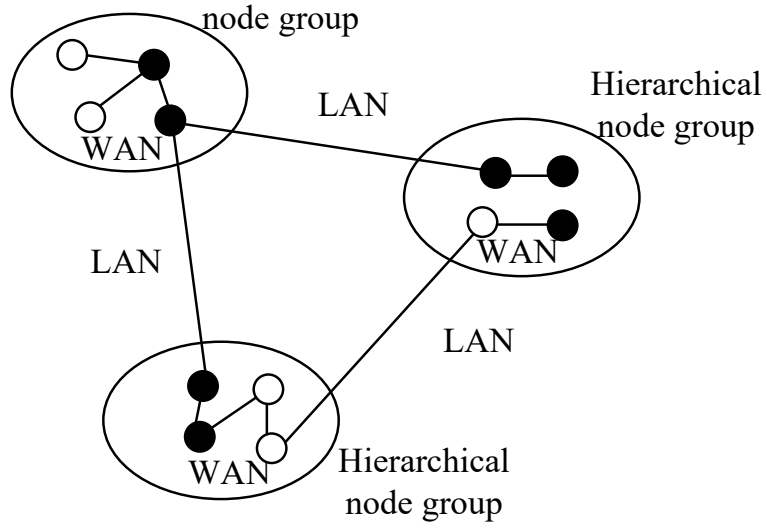

Fig. 1 structure of the hierarchical network

The WAN is defined as a specific set of nodes. Generally speaking, the WAN is a geographically close network, and the connections between its internal nodes are closer than the connections between the network nodes; The LAN refers to a specific node that is closely connected to it. The typical characteristics of a layered network are as follows:

First: wireless-oriented, open channels. The layered network is dominated by wireless communication, and its typical characteristics are open channels, limited bandwidth, and vulnerability to attacks, which also makes the existence of communication links closely related to node locations;

Second: layered networking, good scalability. The layered network mostly adopts a hierarchical layered networking mode in which the core network and the access network are separated, which has the advantages of strong survivability and strong scalability;

Third: dynamic reorganization and strong resistance to destruction. The layered network has the ability of self-organization, self-healing, self-recovery, and real-time reorganization, which makes the network more robust and survivable in harsh environments.

It is necessary to establish a network model that includes both network topology information and network dynamic behavior information [8]. To this end, a hierarchical network dynamic mathematical model is defined as:
$K(T)=\frac{\partial \overline{u_{i}}}{\sum N x_{i}}$

where, $K(T)$ represents the multi-hop wireless network; $\sum N x_{i}$ represents the discrete coefficient; $\partial$ is the network node set, including the Mesh nodes and the Adhoc nodes; $\overline{u_{i}}$ represents the spatial location set of the network node.

Network nodes, links, locations, and behaviors are all functions of time, so the network is a dynamic network that changes over time. For a specific network at a given time, that is:

$G=M(t) / \frac{E_{\max }}{p_{i}}$

where, $G$ represents a deterministic quantity; $M(t)$ represents continuous changes in multiple graphs that approximate the network topology change process parameters; $p_{i}$ represents the network speed; $E_{\max }$ represents the hierarchical network topology and location-related characteristic parameters.

\section{Network TOPOLOGY INFORMATION CONTROL AND OPTIMIZATION}

Effective network topology means that the network has good connectivity and integrity. Therefore, network connectivity indicators can be used to measure the effectiveness of the network topology [9]. At present, there is no accepted network connectivity index in academia. Generally, the connection strength is represented by the connectivity. Connectivity refers to the minimum number of nodes (or edges) that need to be removed to make the network disconnected. Connectivity mainly reflects the difficulty of segmenting the network, and cannot reflect the quality of the network connectivity, especially the connectivity after the network is segmented.

\section{A. Nodes in network topology}

With the development of research on the invulnerability of complex networks, a large number of researchers use the maximum connected subgraph size to characterize the attack effect, however, this indicator has the problems of poor representativeness and distinguishing ability [10]. Some researchers use the number of connected branches obtained after the attack to characterize the attack effect, but this indicator cannot reflect the difference caused by the difference in the size of the connected branches. Some scholars use the average value of the shortest path of the network to represent the network connectivity, however, as the attack intensifies, the average shortest path becomes larger and then becomes smaller. Among the existing indicators, relatively reasonable indicators of network connectivity are global efficiency and connectivity coefficients:

$K=\frac{U C_{u}}{Q} \sqrt{\frac{l_{i}}{s t}}$ 
$P=\frac{w C_{u}}{F} \sqrt{\frac{P}{\partial t}}$

where, $K$ represents the total number of network nodes; $\frac{U C_{u}}{Q}$ represents the number of connected branches of the network; $\sqrt{\frac{l_{i}}{s t}}$ represents the number of nodes included in the connected branches; $\frac{w C_{u}}{F}$ represents the average shortest path length of the connected branches; $\sqrt{\frac{P}{\partial t}}$ represents the network connectivity index.

The effectiveness of the network refers to the network where the total social utility is maximized, which can be given as defined below. The same node can build different network structures. The effective network refers to the network that maximizes the network value. In the process of network formation, if a network node in a network graph has the right to form a connection (or edge, the same below) or to interrupt an edge, the connection of edges is formed when both nodes agree.

Because when the node density in the network reaches a certain level, whether from the perspective of information transmission or acquisition, redundant nodes have a very negative impact on the energy consumption of the network. Putting redundant nodes into a sleep state will significantly reduce the energy consumption of the network [11].

\section{B. Connectivity of network topology signals}

The topology control problem is regarded as a network layer problem, and the link detection between neighboring nodes is performed at the network layer without relying on the support of the underlying protocol or geographic information. The transmit power of each node can only be adjusted at a few determined power values and power levels, and the continuous value topology control and route optimization processes cannot be completed in user space [12]. The optimized topology control and routing results are then transferred into the kernel space to control the data forwarding and nodes transmit power adjustment processes.

(a) Wireless channel modeling

Modeling in wireless channels is a complex task. Wireless media is very sensitive to path loss, noise, interference, and blocking caused by physical obstacles. It affects the quality of the received signal and is a function of the propagation distance. Path loss $m k$ is equal to the ratio of received energy to transmitted energy. The calculation formula is:

$$
m k=E \partial / \frac{x}{a}
$$

where, $E$ represents the received signal energy; $\frac{x}{a}$ represents the transmitted signal energy; $\partial$ represents the signal reception quality.

In addition to path loss, the bit error rate in transmission and the quality of the received data are related to the noise power, transmission power, and location of other sending nodes in the vicinity of the node (assuming that all nodes send data on the same frequency band).

Assume that the nodes in the network are evenly distributed in a two-dimensional space. The maximum transmit power of each node is the same as the threshold of the receiver and has the same path loss model. It is assumed that each node can determine the direction of the node sending the message relative to itself when receiving the message, that is, both nodes can know each other's direction when exchanging broadcast confirmation messages. According to the literature, the technique of estimating direction is feasible without position information. Based on the above analysis and the assumptions of the network model, a mathematical model can be designed using more than one directional antenna. The model is divided into three parts:

First, information acquisition. The movement of the nodes causes the network topology to change dynamically. Using the technology of periodic broadcast messages and the direction of the antenna to sense the message, the movement status and relative position information of neighbor nodes in the current one-hop range are obtained, so as to grasp the local network topology information.

Second, construct an approximate graph. After mastering the nodes of the local network topology information, according to the topology edge constraints proposed in the above analysis, the approximate graph parameters are obtained, and the locally optimized network topology is constructed, thereby completing the establishment of the overall network topology approximate graph.

Third, approximate graph parameters. Determine whether there is a self-directed unidirectional link, and adjust the parameters of the approximate graph to make the network topology a symmetric directed graph. At the same time, it is necessary to ensure that the other end node connected to this self-directed unidirectional link achieves bidirectional communication.

Connectivity is also called node connectivity, which expresses the influence of nodes on network connectivity. Connectivity corresponds to the minimum node cut set of the network. In addition, network survivability is an essential aspect of reliable communication services. Survivability not only refers to the robustness of the network when it faces natural failures, unexpected events, unintentional operation errors, or misconfigurations, but it also refers to the robustness of the network when malicious hostilities exist. Network survivability can be reflected by the ability to resist network segmentation [13].

(b) Wireless network communication mode

The factors that cause network segmentation are divided into physical layer factors and link layer factors. The problems of hidden and exposed terminals unique to wireless multi-hop networks can cause packet collisions, which can lead to network segmentation. The hidden terminal is a node that is within the 
communication range of the receiving node and outside the communication range of the sending node. As shown in the figure, node $\mathrm{B}$ is within the communication range of nodes $\mathrm{A}$ and $\mathrm{C}$, but nodes $\mathrm{A}$ and $\mathrm{C}$ are not within the communication range of each other. When $\mathrm{A}$ sends data to $\mathrm{B}$, even if the hidden terminal $\mathrm{C}$ cannot hear the information sent by $\mathrm{A}$, it may send information to $\mathrm{B}$, which will cause the message to collide at the receiving node $\mathrm{B}$ and cause $\mathrm{B}$ to fail to parse any information correctly. This reduces the channel utilization and increases system latency. In this case, the receiving node $\mathrm{B}$ cannot establish a communication connection with $\mathrm{A}$ and $\mathrm{C}$, and cannot even communicate with all other nodes in the network, which forms an orphan node and causes network division. Orphan nodes are an extreme case of network segmentation. The hidden terminal diagram is as shown in Fig. 2.

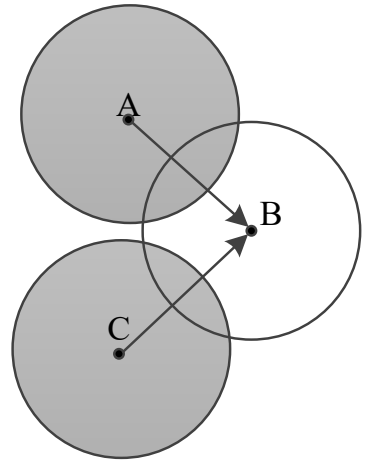

Fig. 2 schematic diagram of hidden terminal

The exposed terminal is a node that is within the communication range of the sending node and outside the communication range of the receiving node. As shown in the figure, when node B sends information to $\mathrm{C}$, node A delays sending because it hears B's transmission, but $\mathrm{A}$ is outside the communication range of $\mathrm{C}$. As long as it does not communicate with B, its transmission will not affect B. At this time, A cannot communicate with any node. In this case, the exposed terminal will become an orphan node and cause network division.

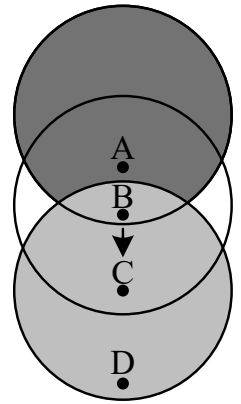

Fig. 3 schematic diagram of exposed terminal

The topology evolution of multi-hop wireless networks in offensive and defensive scenarios is mainly realized as changes in nodes and links, including node movement and increase and decrease, link increase and decrease, and reconnection [14].

Node moves. Mobility is an important feature of multiple wireless networks. Due to the restriction of radio wave propagation, when a node moves away from another node or near its node, the connection relationship between the nodes will change, thereby changing the network topology.
Node increase or decrease. The addition of new nodes, the activation of standby nodes, and the recovery of failed nodes will cause network nodes to increase and expand. Node withdrawal, damage, failure, etc. will cause network nodes to decrease and scale down. The increase or decrease of nodes is an important form of network topology evolution [15].

Increase and decrease of links and reconnection. Setting up a new link, enabling a backup link, etc. will cause an increase in network links. Link interruptions and failures can reduce network links. Changing the link endpoint will cause the link to reconnect. Link additions, deletions, and reconnections are important manifestations of network topology evolution [16].

The same subnet node priority connection strategy. Priority is given to rebuilding the local area network, and the nodes in the subnet are preferentially connected [17].

Orphaned node priority connectivity policy. To avoid islands of information, the communication links of isolated nodes are restored first. At present, the research on network topology reconstruction strategies is extremely limited. In order to enhance network robustness, it is urgent to strengthen the research on network optimization and repair strategies.

\section{Topology reconstruction strategy}

The initial phase of the algorithm is called the information collection phase. In this phase, each node broadcasts messages at the maximum transmit power to learn about neighbors and topology information within a hop range. The specific steps for information collection are as shown in Fig. 4.

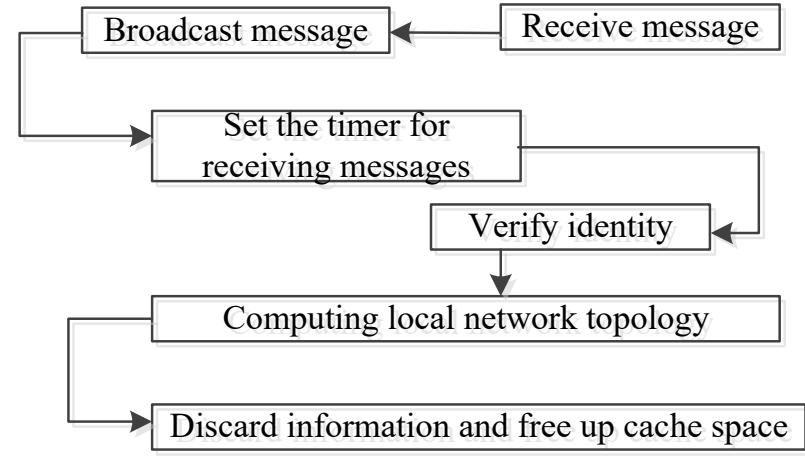

Fig. 4 information collection steps

In order to adapt the algorithm to the dynamic changes of the network topology, the information collection process is performed periodically with the broadcast of messages. During the next cycle of information collection, the nodes work with the adjusted power. To avoid disrupting network connectivity, when it is found that the network is about to split, the power is given a certain increase if the number of neighbors is less than the set threshold [18] [19].

After all nodes in the network perform the information collection process, the local topology information obtained is calculated and judged. Since the maximum transmit power of each node is the same, the original network topology is a symmetric directed graph, and the approximate graph generated is a directed graph. The process of obtaining an approximate graph is as shown in Fig. 5. 


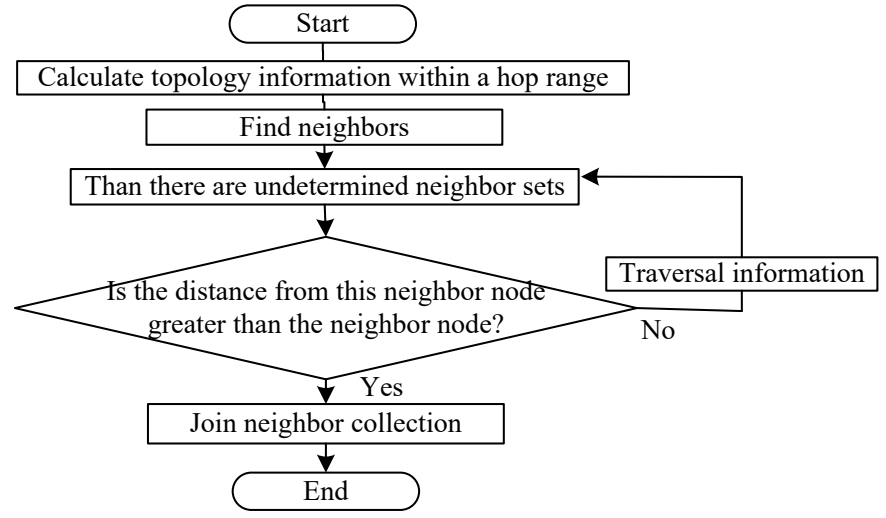

Fig. 5 the approximate graph generation process

First, the introduction of the symmetric mechanism does not consider unidirectional links. There may be unidirectional links in the network topology determined according to the approximate graph generation rule, which results in the failure of normal communication between nodes to affect network connectivity and other performance. The premise of network topology control is to ensure the connectivity of the network. The use of symmetric mechanisms can ensure the connectivity of the network, thereby ensuring the performance of the network in other aspects. If the network topology is not a symmetric directed graph, you can also ensure that the network is connected by changing the routing strategy [20]. However, current layer protocols only consider bidirectional links, and many routing protocols designed for networks can only use unidirectional links. Therefore, building a connected network consisting of bidirectional links is necessary for the response, mechanism, and transmission and retransmission of packets at the link layer.

\section{The establishment of two way link connection network}

(a) Establishment of hierarchical mathematical model

There are two main methods to ensure that the links in the network are bidirectional links, and a mathematical model of the hierarchical network topology is established. This model uses the position parameters of the hierarchical network nodes to reflect the topology and location-related characteristics of the multi-hop wireless network. The network behavior parameters are used to describe the evolution rules and algorithms of the network and describe the dynamic behavior of the network. From the perspective of hierarchical network topology, network behavior parameters can be used to describe the increase or decrease of network nodes, failure or resurrection, and location movement, as well as the increase, decrease, disconnection, or reconnection of network links. Therefore, this model can effectively describe the structural and behavioral characteristics of hierarchical line networks, and can be used to study the topology evolution of multi-hop wireless networks.

It is assumed that after the network is formed, there are three main changes in the evolution process: new nodes are added, the original nodes are withdrawn, and the network structure is optimized and reorganized. In the process of network evolution, the network can be regarded as a gradual evolution. Each node is interrupted at each step when it breaks an existing edge or joins a new edge with other nodes. It can only interrupt one edge or join a new edge at a time. That is, he does not interrupt or join two or more edges at the same time.

The layered model was shown in Fig. 6.

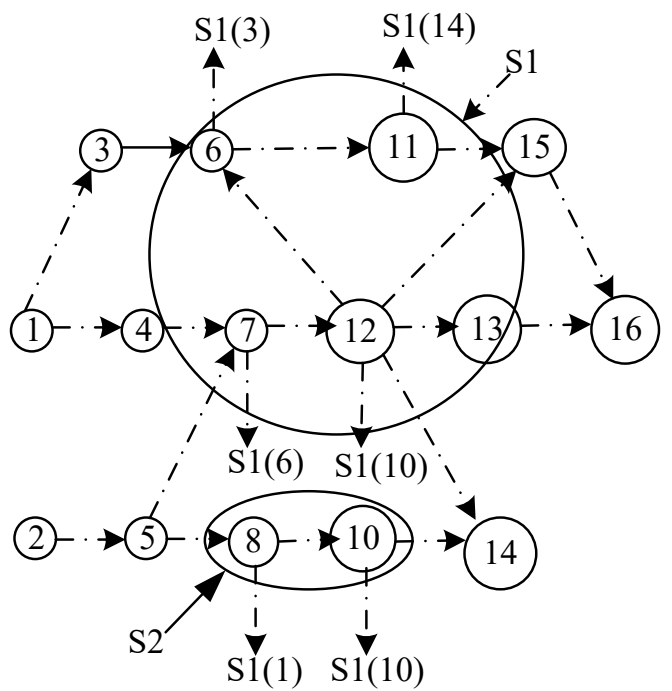

Fig. 6 layered model

where, S1(3) represents the nodes in the second-layer subnetwork S1, the S2(1) generation represents the subnetwork, and the rest of the nodes in S2 are similar.

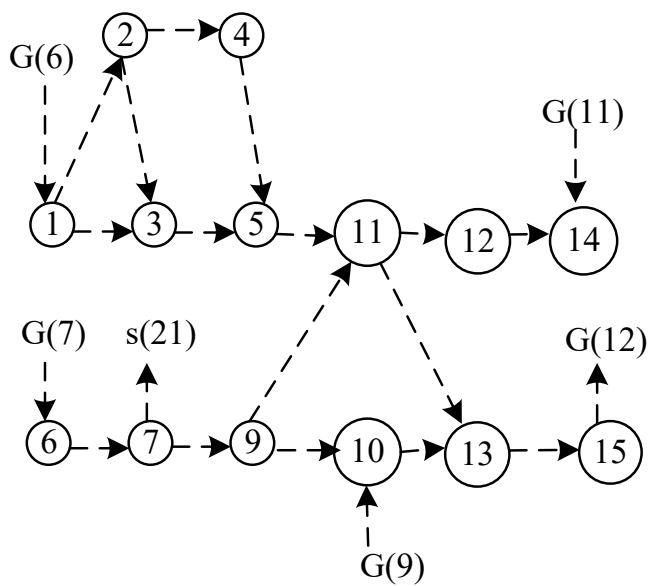

Fig. 7 structure of the first layer network

where, $G(6)$ represents the total network, the nodes in $G$ represent the third-level sub-network nodes, and the rest are similar.

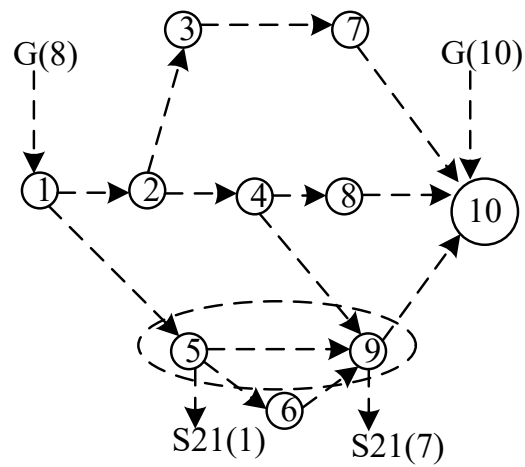

Fig. 8 structure of the second layer network 


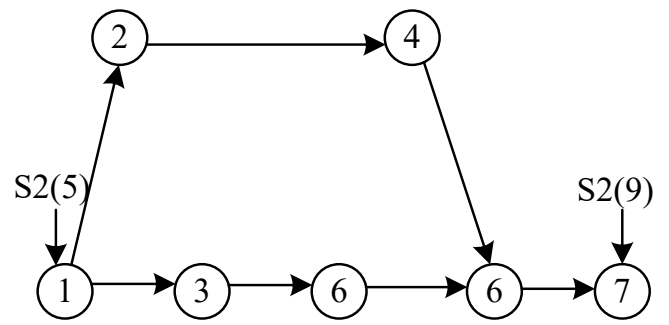

Fig. 9 structure of the third layer network

First, all unidirectional links need to be removed to provide a relatively simple topology, which improves the effectiveness of multiplexing;

Second, the unidirectional link is changed to a bidirectional link, which provides more routing options. Weighing two methods, the latter is selected in the symmetric mechanism mainly for the following three reasons:

1) Less weakening of network survivability;

2) No need to change the routing protocol selection principle;

3) The symmetry mechanism that changes a unidirectional link to a bidirectional link is convergent.

(b) Transition from unidirectional link to bidirectional link

Although the network topology determined according to the approximate graph generation rules is a connectivity graph, there may be unidirectional links, which causes nodes to fail to communicate properly, which affects logical network connectivity and even other network performance. Therefore, consider changing the unidirectional link in the network into a bidirectional link, which not only solves the connectivity problem, but also provides more options for routing. Proper redundancy can improve the robustness and survivability of the network, and reduce the probability of network segmentation due to link failure.

Before the node adjusts its power, in order to avoid generating a one-way link to itself, it can determine to delete the neighbor after receiving the "agree" message, otherwise it is determined that the node is a neighbor, and then the node determines the transmit power according to the final set of neighbors determined. Thus, the purpose of making the unidirectional link in the approximate figure a bidirectional link is achieved, and the node transmit power is as shown in Fig. 10.

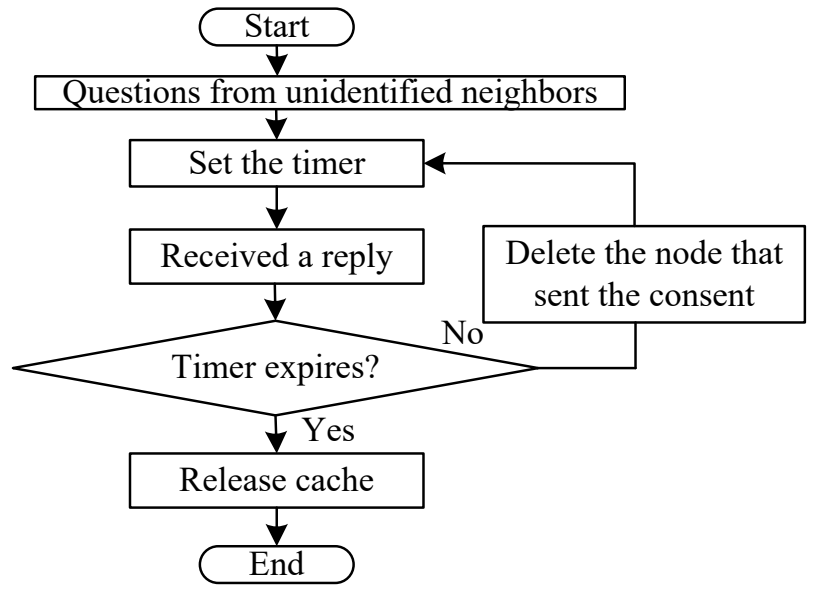

Fig. 10 adjustment process of transmit power
The invulnerability of the network means that at least a few nodes or links need to be destroyed in order to interrupt the communication between some nodes, that is to say the difficulty of destroying a network. This is explained from the perspective of topology. Invulnerability also includes the reliability of each element in the network. Here, the reliability of an element is defined as the probability that the element works normally. The survivability of the network is proposed from the concept of graph theory, and it is widely used in the reliability analysis of communication networks. Destructiveness describes the impact of network topology on the reliability of a communication network from the perspective of network connectivity, and it is a deterministic measure of reliability. The essence of network survivability is to study the reliability of the network's topological structure, and it is a static indicator of network reliability. The indicators that measure the survivability of the network topology include equilibrium, cohesion, and connectivity. The degree of equilibrium reflects the difference in the importance of nodes in the network. Since the nodes are assumed to be equal in status and equal in importance before and after topology control, it can be considered that the equilibrium does not change before and after topology control. Cohesion is also called link connectivity, which expresses the effect of links on network connectivity. The cohesion corresponds to the minimum link cut set of the network, and its calculation formula is:

$D=q / \frac{w}{j_{i}}$

where, $D$ is the received signal energy; $q$ is the transmitted signal energy; $j_{i}$ is the path loss refers to the ratio of the received energy to the transmitted energy; $w$ is the signal reception quality.

According to the above process, an evolutionary mathematical model of hierarchical network topology is constructed.

\section{DISCUSSION}

In order to verify the validity of the evolutionary mathematical model of hierarchical network topology designed in this paper, experiments were performed. In order to ensure the rigor of the experiment, the traditional model was compared with the model designed this time, and the accuracy of the hierarchical network topology evolution of the two models in describing the dynamic behavior of the network was compared.

\section{A. Experimental platform}

The experimental test is to establish a test network to simulate the network environment. Although the obtained results may be more accurate, the high cost and complexity of construction make it impossible to apply to the network. Network simulation is a network virtualization technology that uses related software technologies to construct network topology, implement network protocols, and measure network performance. It can obtain specific network characteristic parameters, and then research and analyze network performance to achieve the purpose of 
improving network operating conditions. Network simulation has become an important method for network research due to its flexibility, high efficiency, and low cost.

The experimental platform of this test is NS2, and the operating system is windows. In the dynamic environment, the graphical interface test environment using the laboratory graphics library before simulation was used to plot the graphs of each stage of the topology control algorithm. In the case where the nodes are randomly distributed according to uniform probability density, the original network topology map, approximate map, and network topology map after topology control are completed. The experimental environment is shown in Fig. 11.

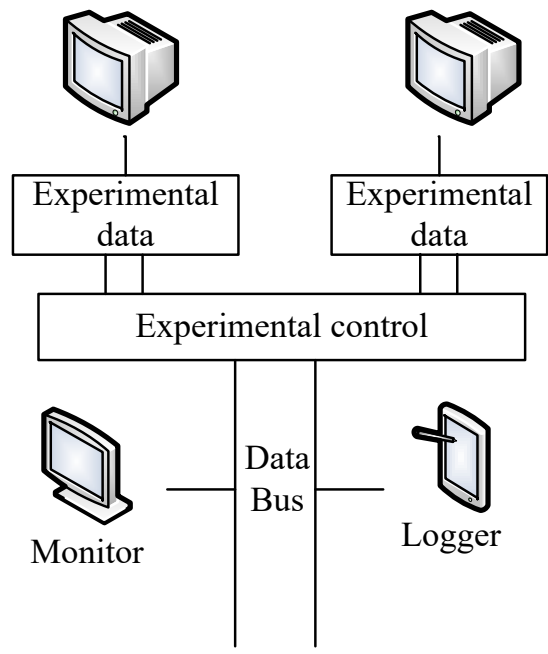

Fig. 11 experimental environment

\section{B. Experimental results}

In order to test the accuracy of the improved mathematical model designed in this paper in describing the dynamic behavior of the network, the running time of the two models is set as 7 minutes. By establishing the test network, the accuracy of the traditional hierarchical network topology evolution mathematical model is compared with the evolutionary mathematical model designed in this paper. The results are shown in Fig. 12.

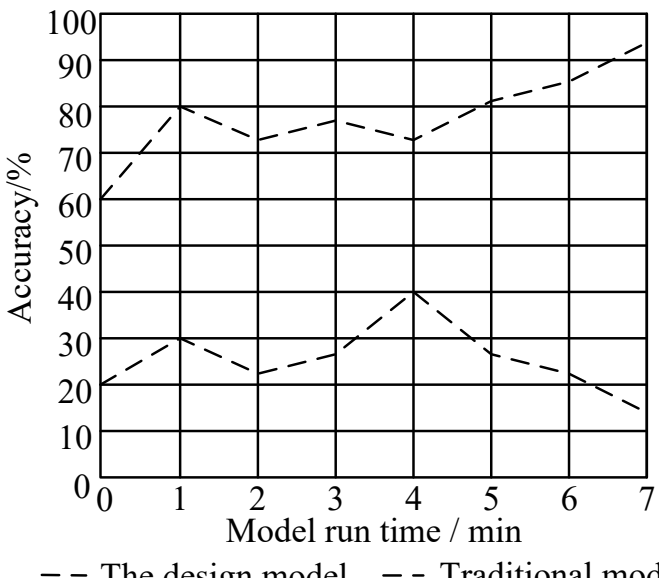

- - The design model - - Traditional model

Fig. 12 experimental comparison results

As can be seen from Fig. 12, when describing the dynamic behavior of the network, the accuracy of the traditional model ranges from $15 \%$ to $40 \%$, and with the change of time, it shows a downward trend, and the accuracy is getting lower and lower; The accuracy of the designed hierarchical network topology evolution model is always above $60 \%$. With the increase of time, the accuracy is also increasing. At the end of the model, the competitive answer is as high as $94 \%$, which is far higher than the traditional model. It can be seen that the topology reconstruction strategy designed in this paper has good accuracy and effectiveness in practical application.

\section{CONCLUSION}

Aiming at the shortcomings of the traditional model, a mathematical model of layered network topology evolution is designed, and the accuracy of the design model is higher than that of the traditional model through experiments, which proves the effectiveness of the model. The establishment of validity index to formulate topology reconstruction strategy can be applied in the future network topology maintenance and segmentation attacks, to provide an effective network topology detection technology for accurate description of the network topology, for scientific research, engineering production technology research and other components of the effective system has important significance.

\section{References}

[1] B. M. Waxman, "Routing of multipoint connections," IEEE J. Select. Areas Commun, no. 9, pp. 1617-1622, 1988.

[2] E. W. Zegura and K. L. Calvert, "A quantitative comparison of graph-based models for Internet topology," IEEE/ACM Transactions on Networking, no. 6, pp. 770-783, 1997.

[3] H. Youssef, S. M. Sait, and S. A. Khan, "An evolutionary algorithm for network topology design," International Joint Conference on Neural Networks, IEEE, pp. 744-749, 2001.

[4] C. Wang, N. Huang, and S. Zhang, "A hierarchical network model for network topology design using genetic algorithm," MATEC Web of Conferences, vol. 119, pp. 01008, 2017.

[5] J. H. Cho and H. Lee, "Dynamic topology model of Q-learning LEACH using disposable sensors in autonomous things environment," Applied Sciences, vol. 10, no. 24, pp. 9037, 2020.

[6] A. Couture, V. François, J.C. Cuillière, and P. Pilvin, "Automatic statistical volume element modeling based on the unified topology model," International Journal of Solids and Structures, vol. 191-192, pp. 26-41, 2020.

[7] Q. Ruhimat, G. W. Fajariyanto, and D. M. Firmansyah, "Optimal computer network based on graph topology model," Journal of Physics: Conference Series, vol. 1211, pp. 012007, 2019.

[8] S. Sarraf, D. D. Desouza, and J. Anderson, "MCADN net: recognizing stages of cognitive impairment through efficient convolutional fMRI and MRI neural network topology models," IEEE Access, vol. 7, pp. 1-1, 2019. 
[9] W. Zeng, D. X. Wang, and J. Y. Chen, "Reliability evaluation of water distribution networks using a valve topology model," Journal of Tongji University (Natural Science Edition), vol. 47, no. 2, pp. 222-227, 2019.

[10]G. Su, Q. Xiong, and Y. Zhang, "Intriguing effects of underlying star topology in Schelling's model with blocks," Physical Review E, vol. 102, no. 1, 2020.

[11]H. L. Ye, J. X. Shen, and Y. K. Sui, "Lightweight topology optimization with buckling and frequency constraints based on independent continuous mapping method," Journal of Solid Mechanics: English Edition, no. 3, pp. 310-325, 2019.

[12] S. Gao, Q. Ye, and L. Liu, "A graphical social topology model for RGB-D multi-person tracking," IEEE Transactions on Circuits and Systems for Video Technology, no. 99, pp. 1-1, 2021.

[13] A. Couture, V. Franois, and J. C. Cuillière, "Automatic statistical volume element modeling based on the unified topology model," International Journal of Solids and Structures, pp. 191-192, 2019.

[14] C. Qian and W. Ye, "Accelerating gradient-based topology optimization design with dual-model artificial neural networks," Structural and Multidisciplinary Optimization, no. 4, pp. 1-21, 2020.

[15] G. Zheng, Q. Xu, and D. Wang, "Distributed topology identification information model of distribution network based on IEC61850," IOP Conference Series Materials Science and Engineering, vol. 563, pp. 042035, 2019.

[16] C. Ren, H. T. Min, and T. F. Ma, "Efficient structure crash topology optimization strategy using a model order reduction method combined with equivalent static loads: Proceedings of the Institution of Mechanical Engineers," Part D: Journal of Automobile Engineering, vol. 234, no. 7, pp. 1897-1911, 2019.

[17] K. Kumar, K. As, and S. S. Kumar, "Stability analysis of solar assisted dc-dc y-source boosting topology using state space model," Sylwan, vol. 163, no. 12, pp. 550, 2019.

[18] J. Miao, "Exploration on extracting geometric model by topology optimization design," IOP Conference Series Materials Science and Engineering, vol. 772, pp. 012072, 2020.

[19]S. Zhang, H. Li, and Y. Huang, "An improved multi-objective topology optimization model based on SIMP method for continuum structures including self-weight," Structural and Multidisciplinary Optimization, vol. 63, no. 2-4, 2021.

[20] G. Krooshof, R. Tuinier, and G. D. With, "Dispersion activity coefficient models. Part 3: A topology preserving group contribution model," Fluid Phase Equilibria, no. 4, pp. 113097, 2021.

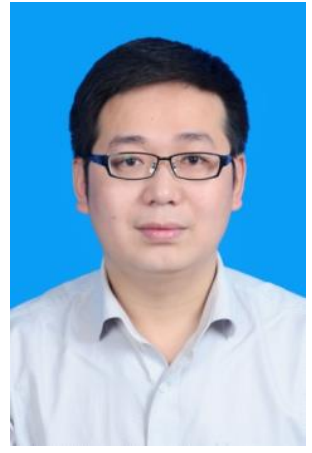

Min Yang, male, was born in May, 1981. His title is professor. He graduated from Panzhihua University in 2005, major in Computer Science and Technology, with a baccalaureate. He graduated from Chongqing University in 2013, major in control engineering, with a master's degree. Now he is working in Dean's Office, Chongqing City Vocational College. He mainly engages in the research of Application of big data technology. So far, he has published 5 EI, CSSCD and CNNC academic articles, presided over 2 provincial and ministerial scientific research projects, participated in 6 provincial and ministerial scientific research projects and authorized 5 patents.

\section{Author Contribution:}

Min Yang: This paper analyzes the hierarchical network, establishes the effectiveness index of topology, formulates the strategy of topology reconstruction, realizes the evolution of hierarchical network topology, and completes the design of mathematical model. The author analyzed and did the experiment and wrote the manuscript.

\section{Creative Commons Attribution License 4.0 (Attribution 4.0 International, CC BY 4.0)}

This article is published under the terms of the Creative Commons Attribution License 4.0

https://creativecommons.org/licenses/by/4.0/deed.en_US 